Portland State University

PDXScholar

Electrical and Computer Engineering Faculty

Publications and Presentations

3-1-1989

\title{
Pump pulse effects in synchronously pumped mode- locked dye lasers
}

Duncan Leo MacFarlane

Lee W. Casperson

Portland State University

Follow this and additional works at: https://pdxscholar.library.pdx.edu/ece_fac

Part of the Electrical and Computer Engineering Commons

Let us know how access to this document benefits you.

\section{Citation Details}

D. L. MacFarlane and Lee W. Casperson, "Pump pulse effects in synchronously pumped mode-locked dye lasers," J. Opt. Soc. Am. B 6, 292-299 (1989).

This Article is brought to you for free and open access. It has been accepted for inclusion in Electrical and Computer Engineering Faculty Publications and Presentations by an authorized administrator of PDXScholar. Please contact us if we can make this document more accessible: pdxscholar@pdx.edu. 


\title{
Pump pulse effects in synchronously pumped mode-locked dye lasers
}

\author{
D. L. MacFarlane and Lee W. Casperson \\ Department of Electrical Engineering, Portland State University, Portland, Oregon 97207
}

Received July 29, 1988; accepted October 11,1988

\begin{abstract}
We consider the role of the pump pulse in the output of a synchronously pumped mode-locked dye laser. Our theoretical formalism is a nonlinear dynamical model that includes semiclassical effects, dipole orientation, and intraband relaxation. In particular, we study the shortening of the output pulse with decreasing pump pulse length and increasing pump level. Both of these predictions agree with experiments.
\end{abstract}

\section{INTRODUCTION}

The synchronously pumped mode-locked (SPML) dye laser has benefited repeatedly during its 20-year history from improved pump sources. The SPML concept was first demonstrated in 1968, when pulsed pump sources were used ${ }^{1-3}$; however, the technique remained of limited practical value until cw mode-locked pump sources were exploited. ${ }^{4,5} \mathrm{Re}$ cently there have again been significant improvements in available pump sources for SPML dye lasers; notable is the fiber-grating compressed Nd:YAG laser, which can deliver subpicosecond pulses. ${ }^{6}$ When pulses from these laser systems are doubled and used for synchronous pumping of organic dye lasers, tunable pulses of 200-300 fsec are obtained directly from the SPML laser. ${ }^{7,8}$ The purpose of this paper is to support these ongoing technological improvements in SPML pump sources with a sound theoretical discussion of the role of the pump pulse in SPML dye lasers. Our numerical results are in good agreement with the recent experiments but suggest that only limited further improvements may be possible.

The theoretical understanding of SPML lasers has advanced on both the analytic and the numerical levels. Because of the stringent approximations imposed in the analytic theory, the two approaches are perhaps best considered distinct, at least in their goals. The analytic work has evolved from the self-consistent pulse picture of Kuizenga and Siegman ${ }^{9}$ and of Haus, ${ }^{10}$ and specific application of this formalism to SPML systems has been considered by Frigo et al., ${ }^{11}$ Ausschnitt et al., ${ }^{12}$ and Kim et al. ${ }^{13}$ These studies have provided closed-form, approximate expressions for the output-pulse characteristics as functions of cavity detuning, system gain, and several dye parameters. More rigorous numerical modeling of SPML dye lasers has been undertaken by many groups, ${ }^{14-25}$ since only this technique facilitates the study of transient effects and specific pulse-shape information. The numerical models also have the advantage that their framework can easily support the study of random noise sources, such as spontaneous emission, which can lead to profound effects at large cavity mismatches far from the optimum cavity length. ${ }^{20,21}$ Much of this computational work has been done in the rate-equation limit; however, semiclassical effects have been shown to be significant in the steady-state pulse shape..$^{21-25}$
An important point of study for nearly all these references has been the dependence of the SPML laser output on the cavity length mismatch between the pump laser and the SPML laser since this is a strong and sensitive test of a candidate theory and is of great practical import. A bandwidth-limiting tuning element is found in most practical systems, and the effects of these filters have been well considered. ${ }^{11-13,16,20,21,25}$ On the other hand, only cursory attention has been paid to the adjustable parameters associated with the pump source. The analysis of Ausschnitt et al. predicts that at zero mismatch the SPML pulse width varies as the square root of the pump pulse width. ${ }^{12} \mathrm{Kim}$ et al., in an analysis under slightly different conditions, show that the leading term of the SPML pulse width goes as the cube root of the pump pulse width. ${ }^{13}$ Both of these findings were highly approximate, and there has been no rigorous numerical study detailing pump pulse effects in SPML lasers.

In this paper we explicitly consider the role of the pump pulse in the output of a SPML dye laser by numerically modeling the Rhodamine $6 \mathrm{G}$ laser under different pumping conditions. In Section 2 we review the theoretical model developed in Ref. 24. This nonlinear dynamical model includes semiclassical effects, dipole orientational effects, and intraband relaxation. The results of our calculations are then presented in Section 3 . In particular, we find a shortening of the output pulse with decreasing pump pulse length or increasing pump pulse level. The effect of pump pulse width is compared with the experiments of Refs. 7 and 8, and the effect of pumping level is tested with our own data. Our theory agrees quantitatively with available experimental data, and we also present results from regimes outside the realm of current experimental interest. In Section 4 we reduce our model to the rate equation limit and discuss the importance of coherence effects in ultrashort-pulse laser modeling. We present a summary in Section 5 and also consider the possibility of further enhancements in SPML lasers.

\section{THEORY}

Our starting point is the semiclassical dye-laser amplifier model developed in Ref. 24. Beginning with the density matrix equations and Maxwell's equations, that paper de- 
rived a set of ordinary, nonlinear differential equations that govern the interplay of the electric field with the molecular populations and the polarization. When the electric field is normalized to its steady-state gain saturating value, this set of equations may be written as

$$
\begin{aligned}
\mathrm{d} D / \mathrm{d} t= & -1 / \tau_{2}\left[\left(1+\tau_{2} / 2 \tau_{1}\right) D+\left(1-\tau_{2} / 2 \tau_{1}\right) M\right. \\
& \left.+2 Q A x-P x^{2}\right] \\
\mathrm{d} M / \mathrm{d} t= & -1 / \tau_{2}\left[-\left(\tau_{2} / 2 \tau_{1}\right) D+\left(\tau_{2} / 2 \tau_{1}\right) M-P x^{2}\right], \\
\mathrm{d} Q / \mathrm{d} t= & -1 / T_{s}(Q-A D x), \\
\mathrm{d} A / \mathrm{d} t= & -L / 2 t_{c} \Delta L\left(A-\int_{0}^{1} Q x \mathrm{~d} x\right) .
\end{aligned}
$$

In Eqs. (1)-(4), $D$ is a normalized population difference, $M$ is a normalized population sum, $Q$ is a normalized polarization, and $A$ is a normalized electric field. The dye decay time from the $S_{1}$ band to the $S_{0}$ band is $\tau_{2}$, and $\tau_{1}$ is the lower manifold intraband vibrational relaxation time. $T_{s}$ is the semiclassical coherence time. In our modeling of the Rhodamine $6 \mathrm{G}$ laser, $\tau_{1}=1 \mathrm{psec}, \tau_{2}=5 \mathrm{nsec}$, and $T_{s}=50 \mathrm{fsec}$. The dynamical rate constant associated with the field is composed of the cavity length $L$, the cavity lifetime $t_{c}$, and the optical length mismatch $\Delta L$ between the pump-laser cavity and that of the dye laser. In this work, $L=1.8 \mathrm{~m}, t_{c}=$ $10 \mathrm{nsec}$, and $\Delta L$ is kept in the $0-30-\mu \mathrm{m}$ range that gives the shortest output pulses. This model is also general enough to include explicitly the orientation of the molecular dipoles, $x$ $=\cos \theta$, and, in particular, we have chosen an isotropic molecular distribution. Thus Eqs. (1)-(3) refer specifically to only one orientational class. The individual classes contribute to the field through the integral over $x$ in Eq. (4). Properly accounting for the different classes is important in accurately establishing the pumping threshold level; misaligned electric dipoles represent an ensemble inefficiency. Orientational effects also manifest themselves in the specific output pulse shape, particularly at small $\Delta L$ 's. ${ }^{24}$

Of specific interest in this paper is the normalized pump function $P(t)$, and for most of this work a Gaussian shape was chosen:

$$
P(t)=P_{0}(2 / \Delta \tau)(\ln 2 / \pi)^{1 / 2} \exp \left[-(2 t / \Delta \tau)^{2} \ln 2\right] .
$$

Importantly from the point of view of this paper, the pump represents two additional control parameters, the pump pulse width $\Delta \tau$ and the pump level $P_{0}$, with which the dyelaser output may be optimized.

It was demonstrated in Ref. 24 that this set of equations adequately describes the pulse shapes for typical SPML dye-laser systems in operation at the time. The main point of comparison in Ref. 24 was the sensitive behavior of the satellite pulses as a function of $\Delta L$, and care was taken to match the experimentally measured autocorrelations with numerically calculated autocorrelations. Missing from our set is a description of the bandwidth-limiting filter with which the operating wavelength is tuned, and it is possible that the agreement between our theory and the experiments below might be improved if the effects of such a filter were explicitly included in our modeling. In Section 3 we present the results of our study, focusing on the importance of $\Delta \tau$ and $P_{0}$ and emphasizing systems of current experimental interest.

\section{RESULTS}

Equations (1)-(4) were numerically solved on a Sun workstation minicomputer, using a standard Runge-Kutta routine for the time integration and a Simpson routine for the integral over the dipole orientation. The individual pulse dynamics has, in general, been reported on extensively, ${ }^{14-25}$ and for this study we are more concerned with the trends associated with various pertinent output pulse parameters as either the pump level or the pump pulse width is varied. In particular we concentrate on the full width at half-maximum (FWHM) pulse width $t_{p}$, the peak intensity $I_{\mathrm{pk}}$, and the time $T_{\mathrm{pk}}$ at which the peak of the output pulse occurs. $T_{\mathrm{pk}}$ is measured with respect to the Gaussian pump pulse,

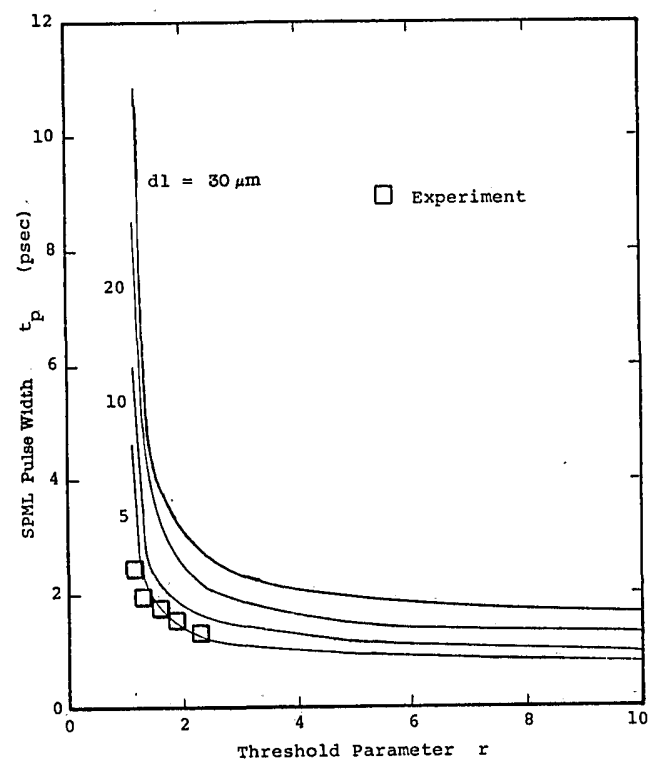

(a)

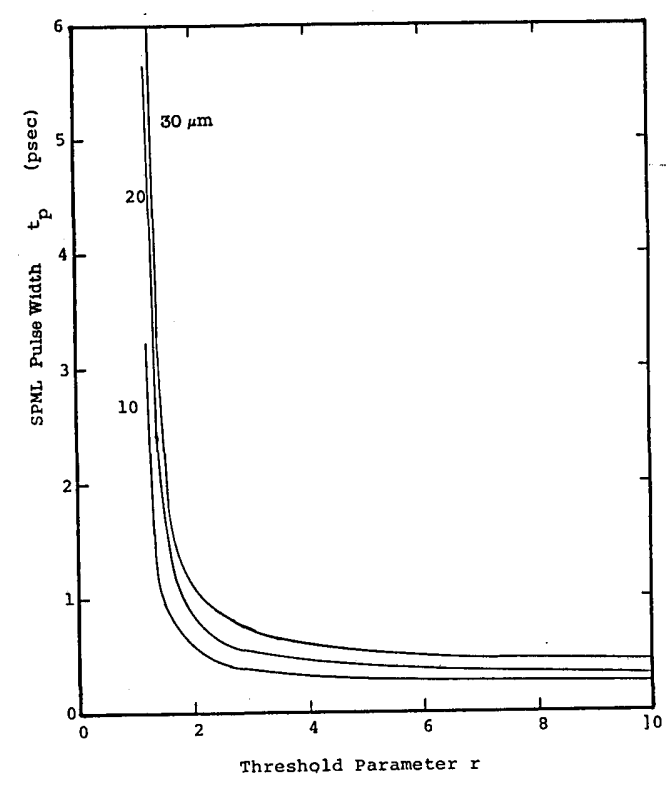

(b)

Fig. 1. SPML pulse width $t_{p}$ as a function of the threshold parameter $r$ at various detunings for pump pulses of (a) $\Delta \tau=100$ psec and (b) $\Delta \tau=10$ psec. The squares in (a) are experimental data points. 


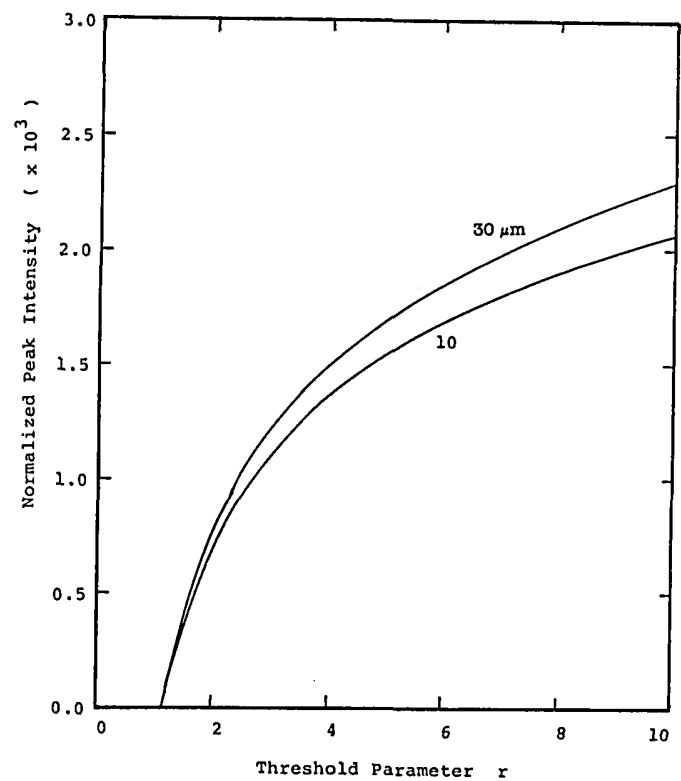

(a)

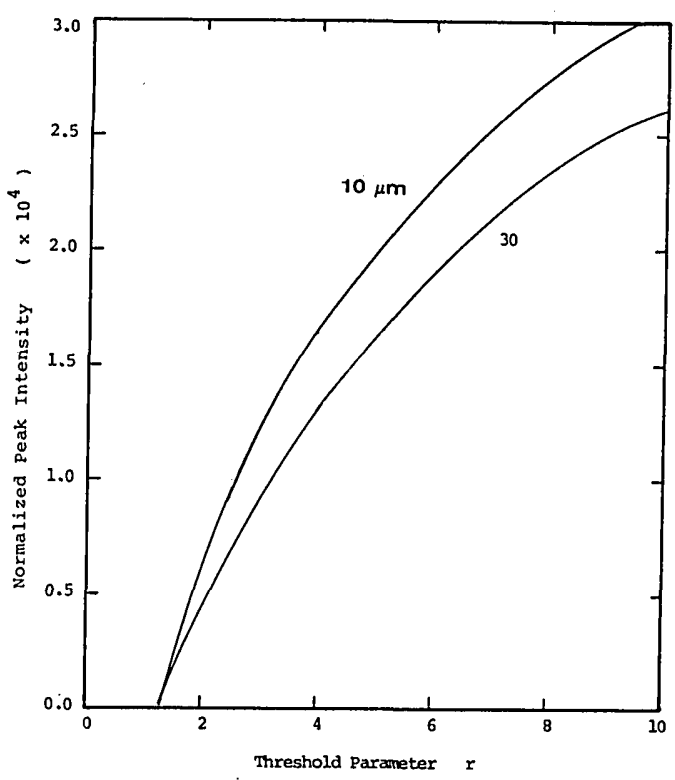

(b)

Fig. 2. SPML peak intensity $I_{\mathrm{pk}}$ as a function of the threshold parameter $r$ at detunings of 10 and $30 \mu \mathrm{m}$ for pump pulses of (a) $\Delta \tau$ $=100$ psec and (b) $\Delta \tau=10$ psec. Noteworthy is the order-ofmagnitude difference in scale between (a) and (b) and the different detuning necessary for optimum peak intensity.

which is centered at zero, and it should therefore be noted that when the dye-laser pulse occurs in the first half of the pumping process, $T_{\mathrm{pk}}$ is negative.

In keeping with our own SPML system, an argon-ionpumped Rhodamine dye laser, and the more recently developed systems in Ref. 8, we studied the effects of the pump level on $t_{p}, I_{\mathrm{pk}}$, and $T_{\mathrm{pk}}$ for pump pulse widths of 100 and 10 psec. The pump level is given here in units of the threshold parameter $r$, which is the pump level divided by the threshold pump level. Hence $r=1$ corresponds to the minimum value for lasing.

Figure 1 shows a rapidly decreasing $t_{p}$ as $r$ increases from 1 to 3 . Values of $r=5$ or higher represent a practically unrealizable region, but increases in $r$ beyond values near 4 result in very small decreases in $t_{p}$. The different contours in Figs. 1 (a) and $1(b)$ correspond to different cavity detunings. The data points in Fig. 1(a) were measured using our commercially available Spectra-Physics argon-ion-Rhodamine 6G dye-laser system. In these experiments, the threshold parameter was varied by linearly attenuating the argon pump beam and was measured as the ratio of the average power of the data point to the average power that just extinguished

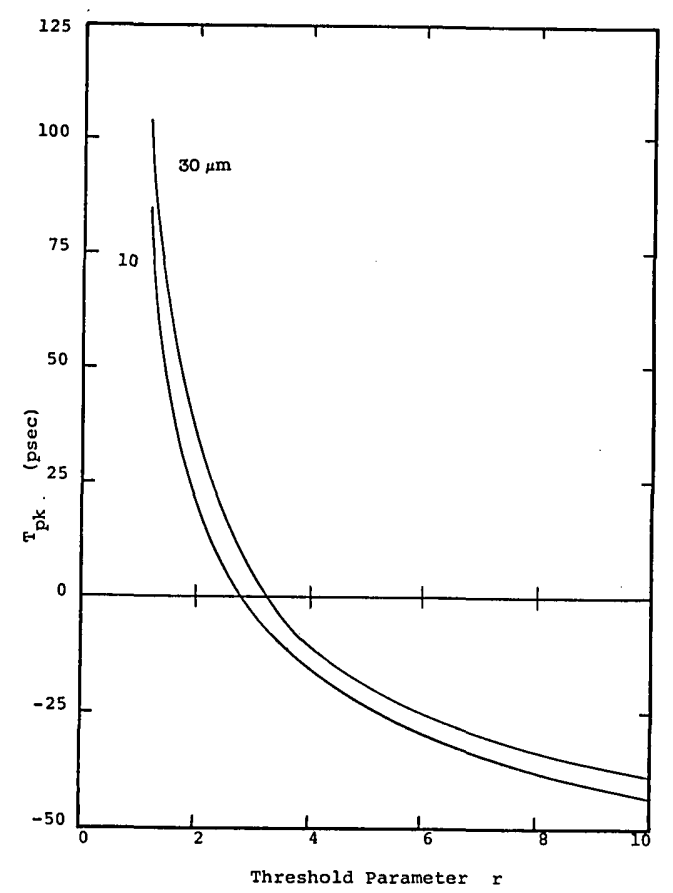

(a)

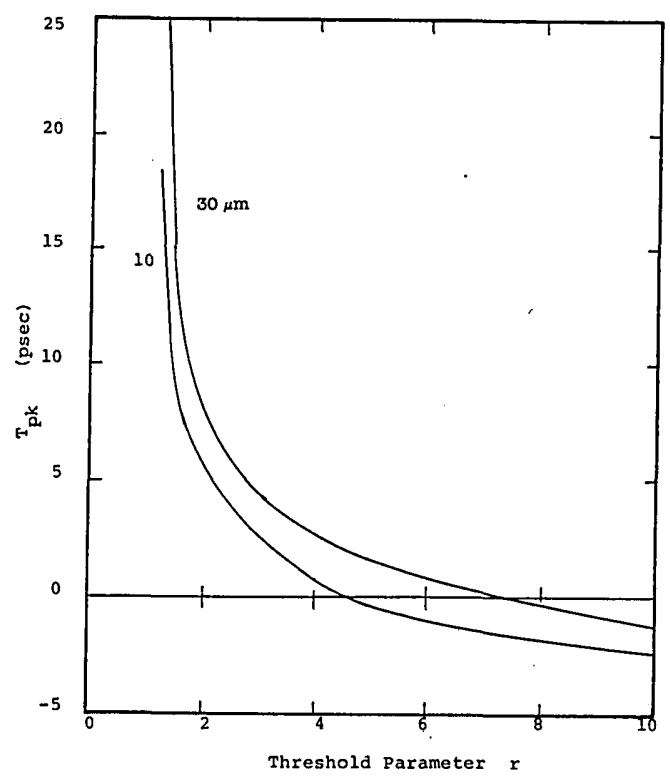

(b)

Fig. 3. Delay time $T_{\mathrm{pk}}$ of the peak SPML output pulse as a function of the threshold parameter $r$ at detunings of 10 and $30 \mu \mathrm{m}$ for pump pulses of (a) $\Delta \tau=100 \mathrm{psec}$ and (b) $\Delta \tau=10 \mathrm{psec}$. The value of $r$ for $T_{p k}=0$ is much higher for the 10-psec pulse. 


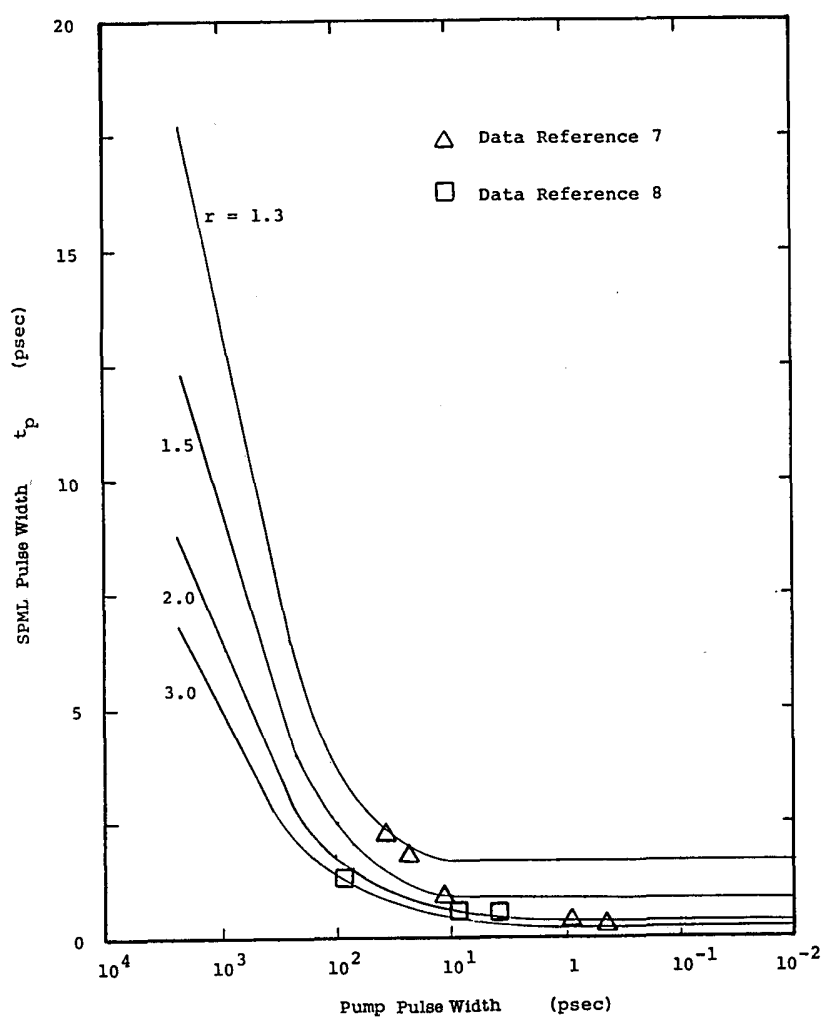

Fig. 4. SPML output pulse width versus $\Delta \tau$ at a cavity detuning of $10 \mu \mathrm{m}$ and for $r=1.3,1.5,2.0$, and 3.0. Superimposed upon this plot are data points taken from Ref. 7 . These are represented by triangles. The rectangles on the graph denote data from Ref. 8 .

the laser. The dye-laser pulse width was measured using a Spectra-Physics 409 autocorrelator at the cavity length that gave the optimum, shortest pulse, which, as suggested by the theory, is $\sim 5 \mu \mathrm{m}$.

In Figs. 2(a) and 2(b) are plots of $I_{\mathrm{pk}}$ versus $r$ for $\Delta \tau=100$ psec and $\Delta \tau=10 \mathrm{psec}$, respectively. Noteworthy is the sharp increase in $I_{\mathrm{pk}}$ as $r$ increases from 1 to 3 . The scale in Fig. 2(b) is 10 times greater than in Fig. 2(a). As $r$ increases for a given $\Delta \tau$ the output pulse occurs sooner, as shown in Fig. 3. The zero crossing for $T_{\mathrm{pk}}$ occurs near $r=3$ for $\Delta \tau=$ $100 \mathrm{psec}$ and between $r=4$ and $r=8$ for $\Delta \tau=10 \mathrm{psec}$.

Of perhaps more interest is the variation in $t_{p}, I_{\mathrm{pk}}$, and $T_{\mathrm{pk}}$ as functions of $\Delta \tau$. Figure 4 is a semilog plot of $t_{p}$ versus $\Delta \tau$ for various values of $r$ and $\Delta L$. Superimposed upon the calculated curves are data taken from Refs. 7 and 8 . While this graph shows significant improvement in pulse width when $\Delta \tau$ decreases from 100 to 1 psec, there is no significant improvement on further shortening, even to 1 fsec. When the midrange values $(\Delta \tau=500$ to $1 \mathrm{psec})$ are fitted to the relation

$$
t_{p}=\beta \Delta \tau^{\alpha},
$$

an $\alpha$ of $\sim 2 / 5$ is typically calculated. This is in fair agreement with the $1 / 2$ value derived by Ausschnitt et al. in Ref. 12 and the $1 / 3$ value derived by Kim et al. in Ref. 13 .

Figure 5 shows the character of $I_{\mathrm{pk}}$ as $\Delta \tau$ is shortened. Of practical interest is the large increase in peak intensity predicted if $r$ is increased to 3 times above threshold and $\Delta \tau$ is decreased to 1 psec, but no improvement in $I_{\mathrm{pk}}$ occurs when $\Delta \tau$ is shortened further. The pump pulse delay $T_{\mathrm{pk}}$ as a

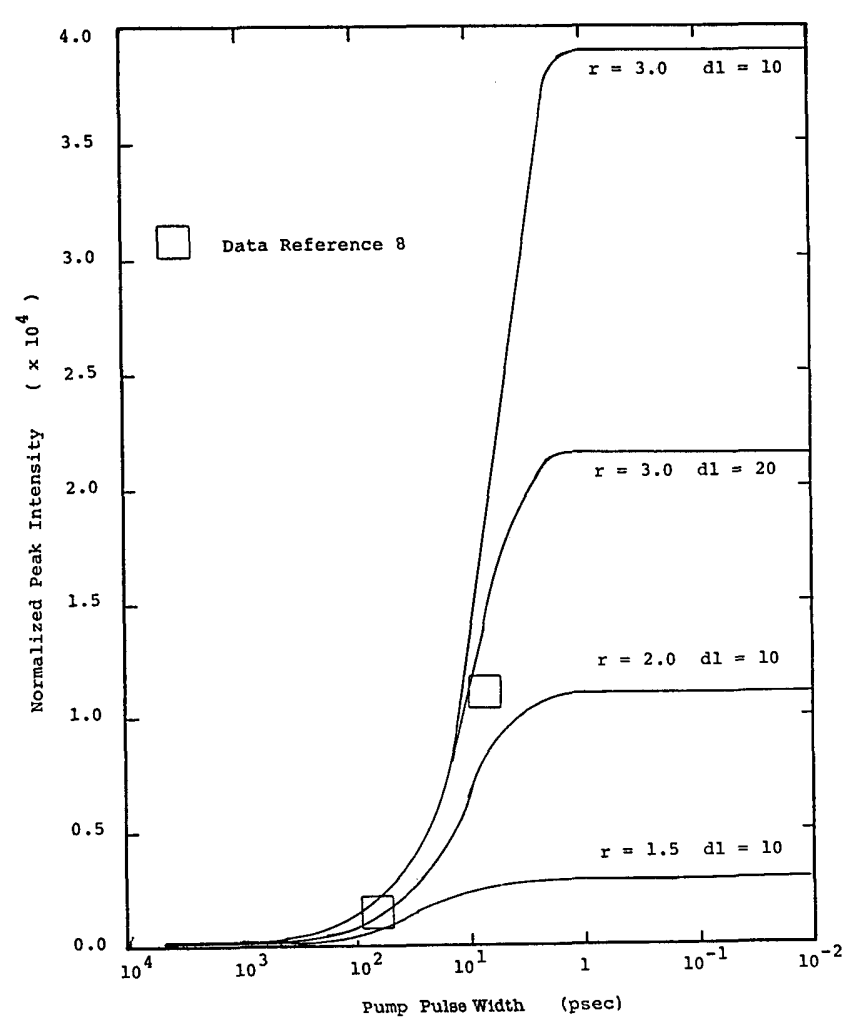

Fig. 5. SPML peak intensity $I_{\mathrm{pk}}$ as a function of the pump pulse width $\Delta \tau$ at various values of $r$ and $\Delta L$. The squares superimposed upon the plot represent data taken from Ref. 8.

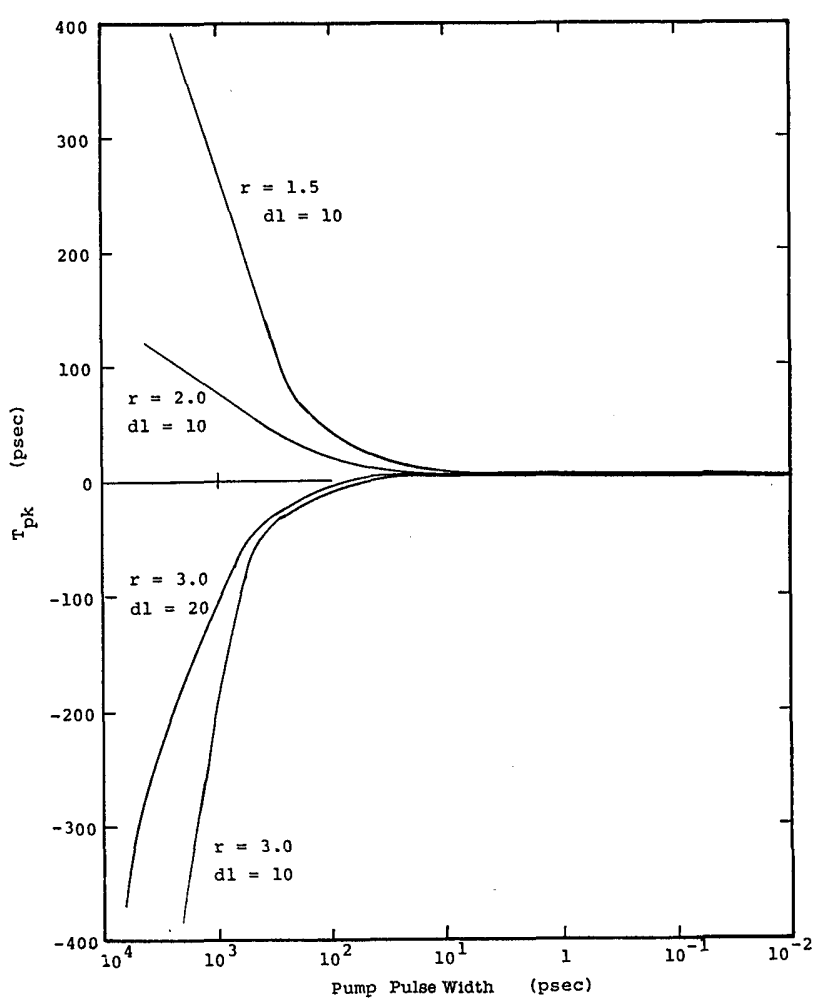

Fig. 6. The SPML pulse delay as a function of the pump pulse width $\Delta \tau$ for various values of $r$ and $\Delta L$. 


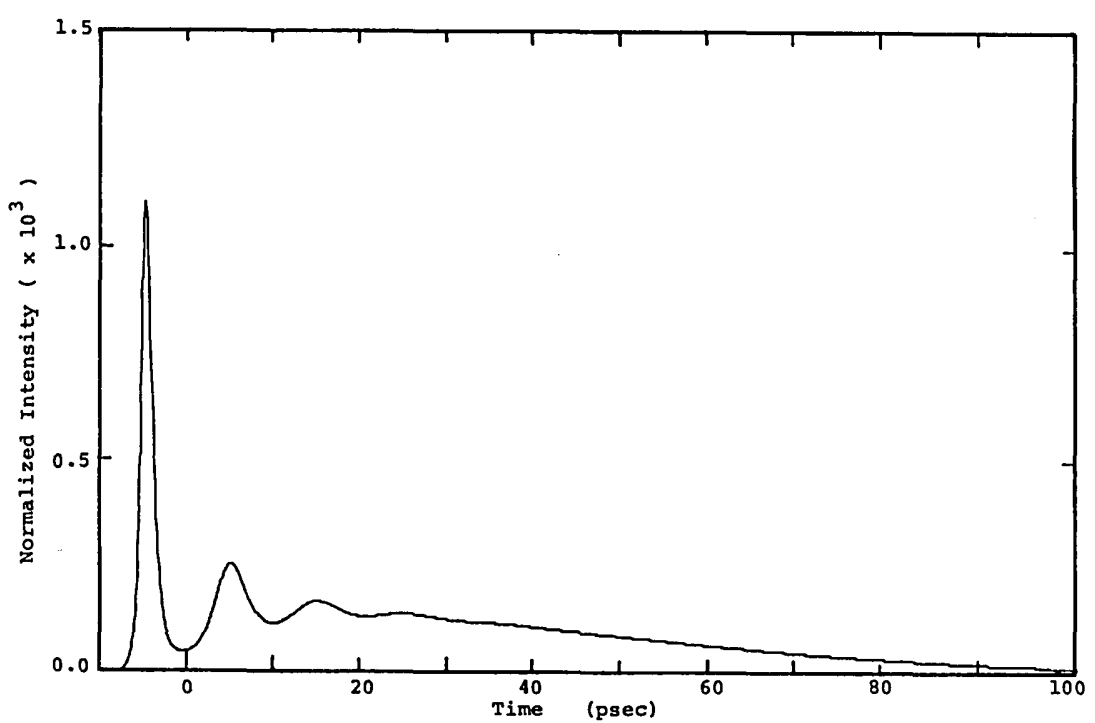

(a)

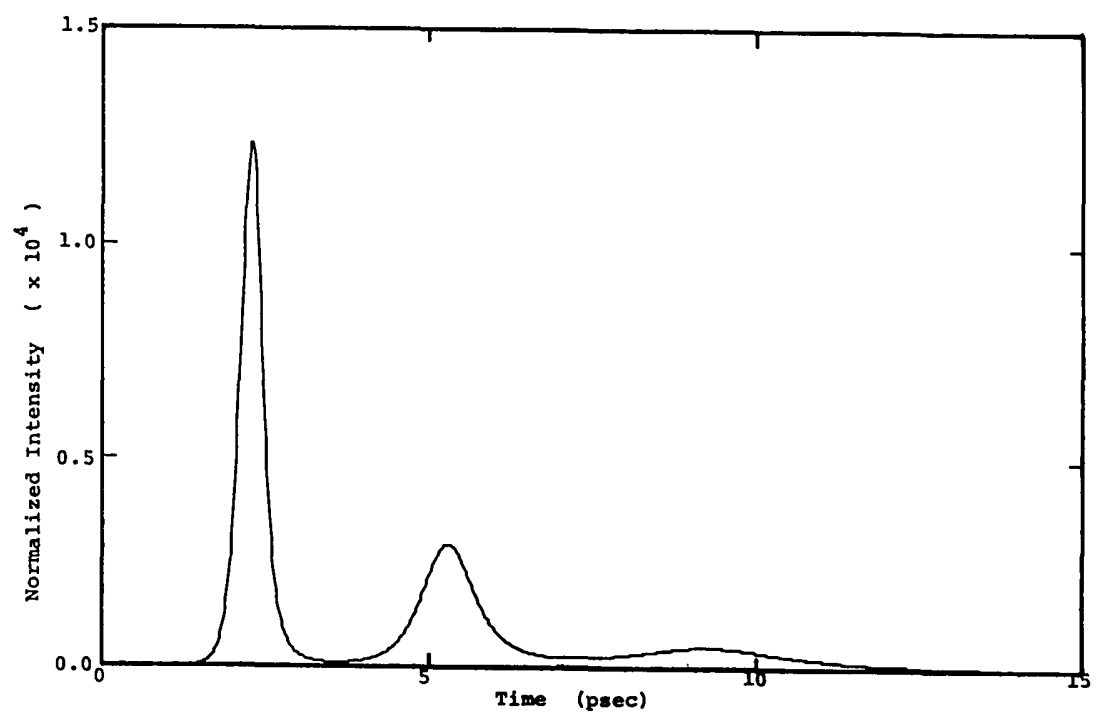

(b)

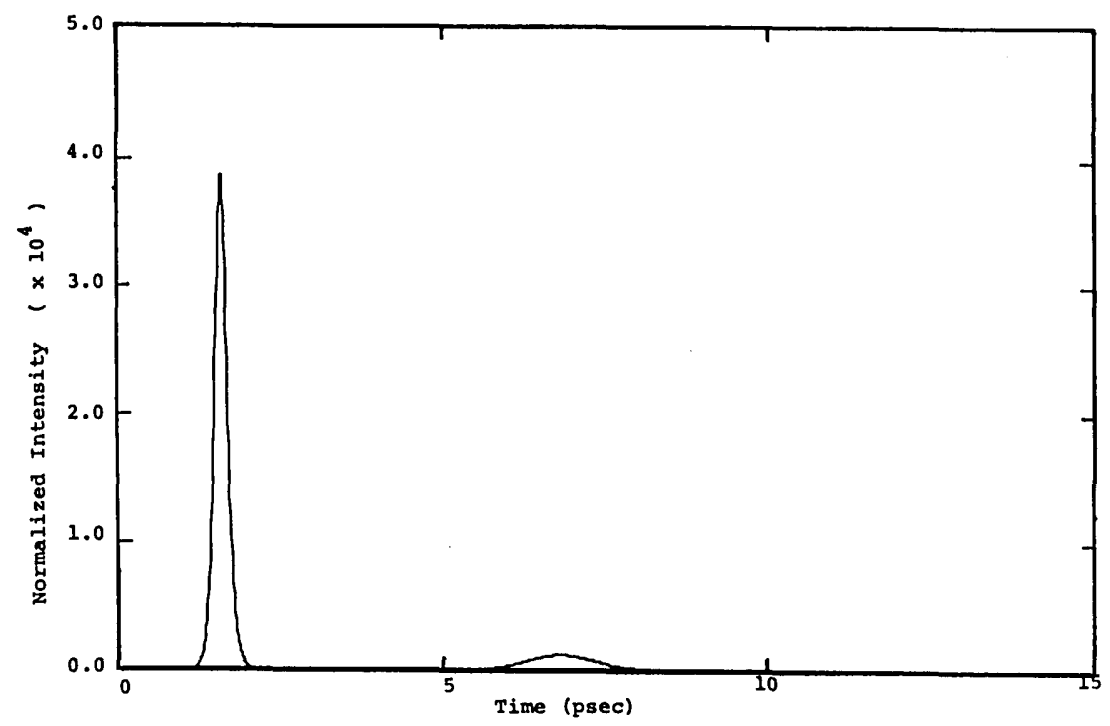

(c)

Fig. 7. SPML output pulses at $r=3.0$ and $\Delta L=10 \mu \mathrm{m}$ for pump pulses of (a) $\Delta \tau=100 \mathrm{psec}$, (b) $\Delta \tau=10$ psec, and (c) $\Delta \tau=1 \mathrm{psec}$. The satellite structure on the trailing edge of the output pulse decreases as $\Delta \tau$ decreases. 
function of $\Delta \tau$ is shown in Fig. 6 for several values of $r$ and $\Delta L$. It is noteworthy that each curve approaches a constant, $T_{\mathrm{pk}}=2.5 \mathrm{psec}$, for $\Delta \tau$ 's shorter than a few picoseconds. Thus, for subpicosecond pump pulses, the dye-laser pulse arrives after the completion of the pump.

This limit where the pulse width $t_{p}$, the peak intensity $I_{\mathrm{pk}}$, and the pulse peak time $T_{\mathrm{pk}}$ become insensitive to further decreases in $\Delta \tau$ is clearly the delta-function limit. For the cases chosen here, this limit is approximately $\Delta \tau=1$ psec.

It is of interest also to study pulse shaping as a function of pump pulse length, and plots of calculated output pulses for fixed $r=3$ and $\Delta L=10 \mu \mathrm{m}$ are shown in Figs. 7(a), 7(b), and 7 (c) for $\Delta \tau=100,10$, and 1 psec, respectively. Importantly, the satellite pulses diminish as $\Delta \tau$ decreases. This is not surprising since the cause of the satellite pulses is principally a surplus of gain delivered by the pump pulse after the primary output pulse is formed. ${ }^{24}$ Near the delta-function limit, all the gain is delivered before dye-laser action starts, and only one pulse is formed. We note that the published autocorrelation in Ref. 8 shows no satellite pulse for $\Delta \tau=3.5$ psec.

Our results so far have been limited to a symmetric Gaussian pump pulse. If one allows for asymmetric pulse shapes, then the rise time and the fall time become decoupled from the pulse width and thus potentially become additional optimization parameters. To test, in particular, the cases of a short rise or fall time with a relatively long pulse time, we used a trapezoidal pump pulse of the form

$$
\begin{aligned}
P(t) & =0 & & t \leq 0 \\
& =P_{0} t / \Delta \tau T_{1} & & 0 \leq t \leq T_{1} \\
& =P_{0} / \Delta \tau & & T_{1} \leq t \leq T_{2} \\
& =\left(P_{0} / \Delta \tau\right)\left(T_{3}-t\right) /\left(T_{3}-T_{2}\right) & & T_{2} \leq t \leq T_{3} \\
& =0 & & 0<t
\end{aligned}
$$

for an extensive series of runs. Here $T_{1}$ is the total rise time, $\left(T_{3}-T_{2}\right)$ is the total fall time, and $\Delta \tau=\left(T_{3}+T_{2}-T_{1}\right) / 2$ is the FWHM pulse length.
After it was verified that the discontinuous nature of this simple shape did not significantly effect the output, a wide range of runs was done with different rise times and fall times. Although it might be plausible to expect that for a given $\Delta \tau$ a shorter rise time would result in a shorter output pulse, we found no trend to support this. Further, in some instances, a shorter rise time resulted in a longer $t_{p}$, a result that in some cases implies that the complicated switching dynamics of a SPML laser depends as much on when the pump pulse turns off as on how fast the energy is delivered. Also of importance is the integrating nature of the gain medium, which comes from its finite response time. Simply put, there are regimes of operation in which the integral of the pump is more important to the output than the form of the pump itself. Consequently it might be only in rare instances that engineered pump pulse shaping becomes important, particularly if this comes at the expense of pump level and overall pump pulse width.

\section{COHERENCE EFFECTS}

An important distinction of the model used here and derived in Ref. 24 is its inclusion of semiclassical effects or, more specifically, in its consideration of a finite coherence time, $T_{s}$ $=50$ fsec. At this value it is reasonable to expect coherence effects to become more pronounced as the system dynamics become faster or, in light of the above results, at high $r$ or small $\Delta \tau$. To test, in general, the importance of coherence effects, several representative runs were taken in the rateequation limit, $T_{s}=0$, for various $\Delta \tau$ 's. The pertinent equations come from the adiabatic elimination of the polarization $Q$ from Eqs. (1)-(4) and are

$$
\begin{aligned}
\mathrm{d} D / \mathrm{d} t= & -1 / \tau_{2}\left[\left(1+\tau_{2} / 2 \tau_{1}\right) D+\left(1-\tau_{2} / 2 \tau_{1}\right) M\right. \\
& \left.+2 A^{2} D x^{2}-P x^{2}\right] \\
\mathrm{d} M / \mathrm{d} t= & -1 / \tau_{2}\left[-\left(\tau_{2} / 2 \tau_{1}\right) D+\left(\tau_{2} / 2 \tau_{1}\right) M-P x^{2}\right] \\
\mathrm{d} A / \mathrm{d} t= & -L / 2 t_{c} \Delta L\left(A-\int_{0}^{1} A D x^{2} \mathrm{~d} x\right) .
\end{aligned}
$$

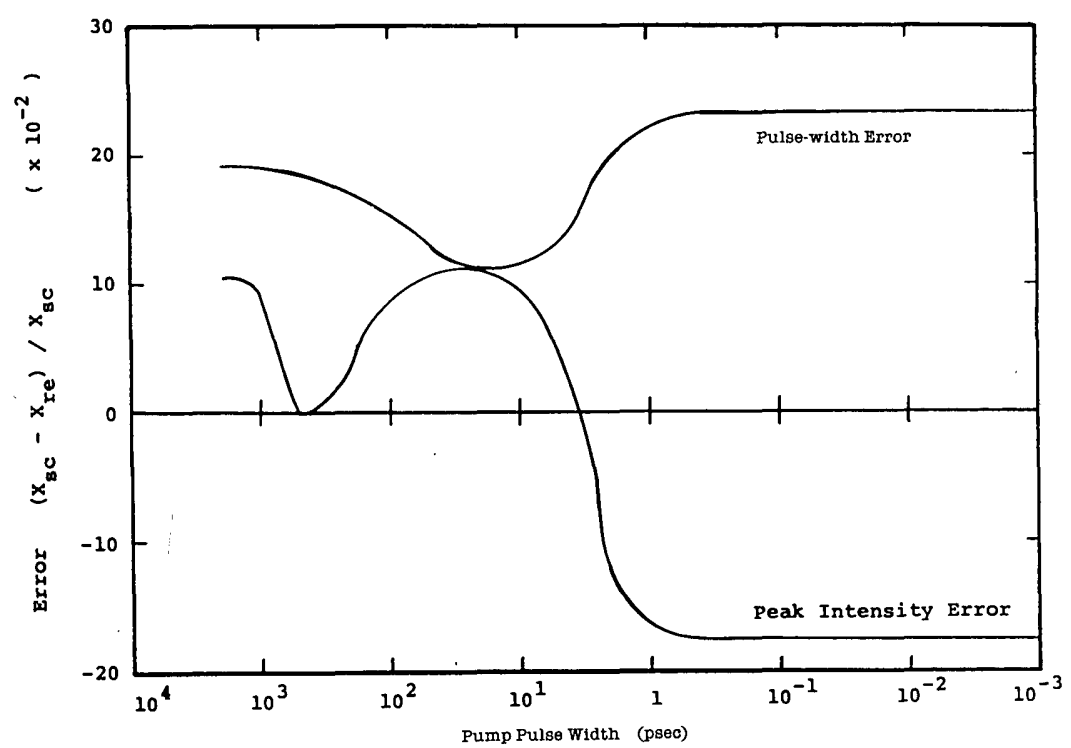

Fig. 8. The error in $t_{p}$ and $I_{\mathrm{pk}}$ that occurs in the rate equation limit as a function of the pump pulse width $\Delta \tau$, shown here for $r=2.0$ and $\Delta L=$ $10 \mu \mathrm{m}$. The error is defined here as the difference between the semiclassical value and the rate equation value, normalized to the semiclassical value. For example, the rate equation error in $t_{\mathrm{p}}$ is $\left(t_{p_{\mathrm{sc}}}-t_{p_{\mathrm{re}}}\right) / t_{p_{\mathrm{sc}}}$. Although the error is small in magnitude, it is a nontrivial function of $\Delta \tau$. 


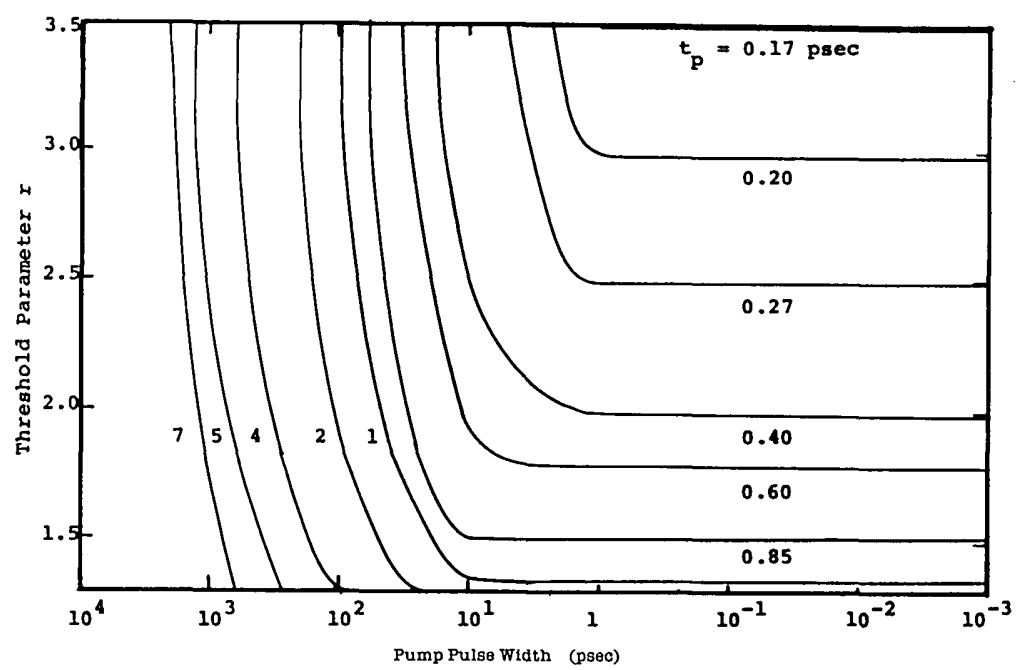

Fig. 9. Contours of constant $t_{p}$ plotted in $r$ and $\Delta \tau$ space. The SPML pulse width $t_{p}$ decreases as either $r$ increases or $\Delta \tau$ decreases but only with diminishing returns.

It was shown in Ref. 24 that coherence effects manifest themselves most significantly in the behavior of the satellite pulses for small detunings. Physically, a finite coherence time implies that the polarization depends on past states of the field, and therefore in the SPML laser case we are, when we include semiclassical effects, carefully modeling the evolution of a trailing satellite pulse as it is acted on by a molecular ensemble dressed by an intense primary pulse. However, we saw above that near the delta-function limit no satellite pulses are formed, and so semiclassical effects in SPML lasers, in one sense, become less important as $\Delta \tau$ is decreased.

A finite coherence time also limits how fast the gain can react, and consequently semiclassical effects also manifest themselves measurably in the character of the primary pulse at all $\Delta \tau$ 's considered. Because of this additional lag, the pulse width $t_{p}$ calculated by using semiclassical effects is always longer than those calculated in the rate-equation limit. For longer $\Delta \tau$ 's the calculated peak intensity is higher when semiclassical effects are included, but for shorter $\Delta \tau$ 's the opposite is true. As is shown in Fig. 8, the rate-equation error in these two quantities, although it is only approximately $20 \%$ on average, is not a simple function of $\Delta \tau$. The additional delay induced by a nonzero $T_{s}$ also implies that $T_{\mathrm{pk}}$ occurs much later in a semiclassical model than in the rate-equation case. For example, at $r=2.0$ and $\Delta L=10 \mu \mathrm{m}$, $T_{\mathrm{pk}}$ in the delta-function limit is $1.5 \mathrm{psec}$ for the rate-equation calculations and $2.5 \mathrm{psec}$ for the semiclassical theory.

\section{CONCLUSIONS}

In this paper we have studied the role of the pump pulse in the output of a SPML Rhodamine 6G dye laser. In particular we focused on two key parameters associated with a Gaussian pump: its width, $\Delta \tau$, and its level with respect to threshold, $r$. Our results for $t_{p}$, the output pulse width, can be summarized in the contour plot of Fig. 9, where lines of constant $t_{p}$ are plotted against $r$ and $\Delta \tau$. As $r$ increases and $\Delta \tau$ decreases, $t_{p}$ gets shorter. The increase in spacing between adjacent curves in the upper right-hand corner represents a case of diminishing returns. For the Rhodamine $6 \mathrm{G}$ laser, state-of-the-art technology is already near this regime, and further shortening of pulses from Rhodamine lasers will most likely come from additional system elements, such as intracavity saturable absorbers and external-cavity fibergrating compressors. On the other hand, we believe that the trends predicted in this paper are general enough to be representative of most SPML systems. Consequently one might expect improvements from SPML lasers operating at different wavelengths as their pump sources mature.

\section{ACKNOWLEDGMENTS}

The authors thank Julie A. Breadleau for her help with some of the computer programming. Conversations with Dae Mann Kim, Andrew Jones, and Nabil Lawandy have been both useful and enjoyable. This research was supported in part by the National Science Foundation and by Tektronix, Inc.

\section{REFERENCES}

1. W. H. Glenn, M. J. Brienza, and A. J. DeMaria, "Mode locking of an organic dye laser," Appl. Phys. Lett. 12, 54-56 (1968).

2. D. J. Bradley and A. J. F. Durrant, "Generation of ultrashort dye laser pulses by mode locking," Phys. Lett. A 27, 73-74 (1968).

3. B. H. Soffer and J. W. Lin, "Continuously tunable picosecondpulse organic-dye laser," J. Appl. Phys. 39, 5859-5860 (1968).

4. C. K. Chan and S. O. Sari, "Tunable dye laser pulse converter for production of picosecond pulses," Appl. Phys. Lett. 25, 403406 (1974).

5. J.M. Harris, R. W. Chrisman, and F. E. Lytle, "Pulse generation in a cw dye laser by mode-locked synchronous pumping," Appl. Phys. Lett. 26, 16-18 (1975).

6. A. M. Johnson, R. H. Stolen, and W. M. Simpson, " $80 \times$ singlestage compression of frequency doubled Nd:yttrium aluminum garnet laser pulses," Appl. Phys. Lett. 44, 729-731 (1984).

7. A. M. Johnson and W. M. Simpson, "Tunable femtosecond dye laser synchronously pumped by the compressed second harmonic of Nd:YAG," J. Opt. Soc. Am. B 2, 619-625 (1985).

8. J. D. Kafka and T. Baer, "A synchronously pumped dye laser using ultrashort pump pulses," in Ultrashort Pulse Spectroscopy and Applications, M. J. Soileau, ed., Proc. Soc. Photo-Opt. Instrum. Eng. 533, 38-45 (1985); technical product information for Spectra-Physics Model 3800 mode-locked Nd:YAG, Model 3695 pulse compressor, and Model 3500 SPML dye laser (1988). 
9. D. J. Kuizenga and A. E. Siegman, "FM \& AM modelocking of the homogeneous laser-part 1: theory," IEEE J. Quantum Electron. QE-6, 694-708 (1970).

10. H. Haus, "A theory of forced mode-locking," IEEE J. Quantum Electron. QE-11, 323-330 (1975).

11. N. J. Frigo, T. Daly, and H. Mahr, "A study of a forced modelocked cw dye laser," IEEE J. Quantum Electron. QE-13, 101109 (1977).

12. C. P. Ausschnitt, R. K. Jain, and J. P. Heritage, "Cavity length detuning characteristics of the synchronously mode-locked $\mathrm{cw}$ dye laser," IEEE J. Quantum Electron. QE-15, 915-917 (1979).

13. D. M. Kim, J. Kuhl, R. Lambrich, and D. von der Linde, "Characteristics of picosecond pulses generated from synchronously pumped cw dye laser system," Opt. Commun. 27, 123-126 (1978).

14. Z. A. Yasa and O. Teschke, "Picosecond pulse generation in synchronously pumped dye lasers," Opt. Commun. 15, 169-172 (1975).

15. A. Scavennec, "Mismatch effects in synchronous pumping of the continuously operated mode-locked dye laser," Opt. Commun. 17, 14-17 (1976).

16. J. Herrmann and U. Motschmann, "Formation of synchronously mode-locked dye laser pulses," Opt. Commun. 40, 379-384 (1982).

17. L. M. Davis, J. D. Harvey, and J. M. Peart, "Rate equation simulation of a synchronously pumped dye laser," Opt. Commun. 50, 49-55 (1984).
18. T. Urisu and Y. Mizushima, "Transient pulse buildup mechanisms in a synchronously-pumped mode-locked dye laser," J. Appl. Phys. 57, 1518-1522 (1985).

19. J. J. Kasinski, L. A. Gomez-Jahn, R. J. D. Miller, P. Geist, B. Geoffrey, F. Heisel, A. Martz, and J. A. Miehe, "Picosecond pulse formation in synchronously pumped dye lasers," J. Opt. Soc. Am. B 3, 1566-1572 (1986).

20. J. M. Catherall and G. H. C. New, "Role of spontaneous emission in the dynamics of mode locking by synchronous pumping," IEEE J. Quantum Electron. QE-22, 1593-1599 (1986).

21. U.Stamm, "Numerical analysis of pulse generation in synchronously mode-locked cw dye lasers," Appl. Phys. B 45, 101-108 (1988).

22. V. A. Nehaenko, "Theory of mode-locked pumping of dye lasers," Sov. J. Quantum Electron. 11, 446-449 (1981).

23. A. I. Kovrigin, V. A. Nekhaenko, S. M. Pershin, and A. A. Podshivalov, "Dynamics of emission from dye lasers pumped synchronously by finite picosecond pulse trains," Sov. J. Quantum Electron. 14, 1346-1353 (1984).

24. L. W. Casperson, "Coherence effects in synchronously pumped mode-locked dye lasers," J. Appl. Phys. 54, 2198-2208 (1983).

25. B. Zandi, L. W. Casperson, and D. L. MacFarlane, "Experimental verification of a semiclassical model for synchronously pumped mode-locked dye lasers with bandwidth limiting elements," in Proceedings of the 14th Congress of the International Commission for Optics (ICO Secretariat, Quebec, 1987). 